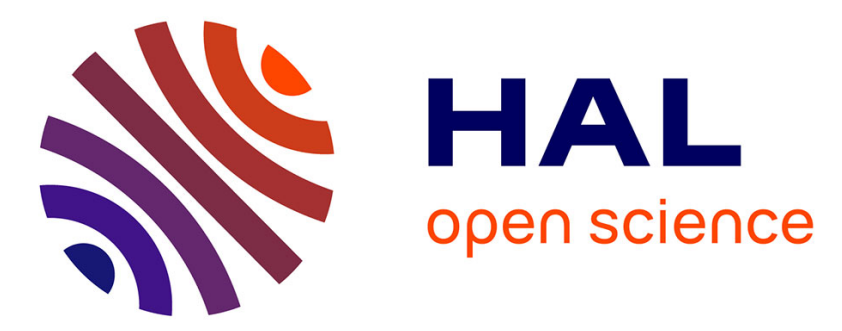

\title{
Converting Water Adsorption and Capillary Condensation in Usable Forces with Simple Porous Inorganic Thin Films
}

\author{
Mickael Boudot, Hervé Elettro, David Grosso
}

\section{- To cite this version:}

Mickael Boudot, Hervé Elettro, David Grosso. Converting Water Adsorption and Capillary Condensation in Usable Forces with Simple Porous Inorganic Thin Films. ACS Nano, 2016, 10 (11), pp.10031-10040. 10.1021/acsnano.6b04648 . hal-01396510

\section{HAL Id: hal-01396510 https://hal.sorbonne-universite.fr/hal-01396510}

Submitted on 14 Nov 2016

HAL is a multi-disciplinary open access archive for the deposit and dissemination of scientific research documents, whether they are published or not. The documents may come from teaching and research institutions in France or abroad, or from public or private research centers.
L'archive ouverte pluridisciplinaire HAL, est destinée au dépôt et à la diffusion de documents scientifiques de niveau recherche, publiés ou non, émanant des établissements d'enseignement et de recherche français ou étrangers, des laboratoires publics ou privés. 


\title{
Converting Water Adsorption and Capillary Condensation in Usable Forces with
}

\section{Simple Porous Inorganic Thin Films}

\author{
Mickael Boudot*1, Hervé Elettro², David Grosso ${ }^{1,3}$ \\ ${ }^{1}$ Sorbonne Universités, UPMC Univ Paris 06, CNRS, Collège de France, UMR 7574, Chimie de la \\ Matière Condensée de Paris, F-75005, Paris, France. \\ ${ }^{2}$ Sorbonne Universités, UPMC Univ Paris 06, CNRS, UMR 7190, Institut Jean Le Rond d'Alembert, F- \\ 75005 Paris, France. \\ ${ }^{3}$ NSE-IM2NP - UMR CNRS 7334, Aix-Marseille Université, Faculté des Sciences de Saint Jérôme, Case \\ 142, 13397 Marseille Cedex 20, France.
}

\begin{abstract}
.
This work reports an innovative humidity-driven actuation concept based on conversion of chemical energy of adsorption/desorption using simple nanoporous sol-gel silica thin films as humidityresponsive materials. Bilayer-shaped actuators, consisting of a humidity-sensitive active nanostructured silica film deposited on a polymeric substrate (Kapton), were demonstrated as an original mean to convert water molecule adsorption and capillary condensation in usable mechanical work. Reversible solvation stress changes in silica micropores by water adsorption and energy produced by the rigid silica film contraction, induced by water capillary condensation in mesopores, were finely controlled and used as energy sources. The influence of the film nanostructure (microporosity, mesoporosity) and thickness and the polymeric substrate thickness, on actuation force, on movement speed and on displacement amplitude are clearly evidenced and discussed. We show that the global mechanical response of such silica-based actuators can easily be adjusted to fabricate tailor-made actuation systems triggered by humidity variation. This study provides insight into hard ceramic stimulus-responsive materials that seem to be a promising alternative to traditional soft organic materials for surface-chemistry-driven actuation systems.
\end{abstract}

Keywords: Sol-gel, humidity, actuator, mesoporous film, silica

An adaptive material or system able to produce mechanical work in response to a specific external stimulus or a change in its environment is the general definition that describes an actuator. Nowadays such materials or systems arouse great interest in many fields as different as materials science, medicine, engineering, robotics, etc. ${ }^{1,2}$

The concepts of man-made actuators rely most of the time on synthetic responsive materials able to convert an energy source into a controlled movement. A wide range of organic and inorganic materials was developed to respond to various types of energy-giving stimuli. For instance, electroactive polymers $^{3,4}$, carbon-nanotube-based structures ${ }^{5,6}$, piezoceramics, ${ }^{7,8}$ or metallic electrochemical thin films ${ }^{9,10}$ to name a few are studied for their response to electric stimuli. Thermoresponsive elastomers, ${ }^{11}$ shape-memory ceramics, ${ }^{12,13}$ and alloys ${ }^{14}$ are known for their ability to convert thermal energy in mechanical work. Concepts that exploit magnetic and light-responsive materials, belonging to the category of pure man-inspired actuators, are also interesting. ${ }^{15,16}$ Another approach, which is inspired by biological systems, consists of mechanical movements generated from chemical energy sources as observed with plants, for example. ${ }^{17,18}$ Synthetic actuation mechanisms driven by either 
gas adsorption, molecule diffusion, or $\mathrm{pH}$ stimulus are several bioinspired examples to turn chemical energy into an artificial mechanical movement. ${ }^{19-22}$ Humidity-responsive actuators, involving pure or composite hygroresponsive polymers, are a particularly active field. ${ }^{23-26} \mathrm{~A}$ fast actuation speed, a large amplitude of movement, and a robust reactivity are the essential requirements for sensitive materials to tailor valuable actuators and create ingenious smart devices as energy generators, ${ }^{19,27}$ artificial muscles, ${ }^{28}$ or programmable origamis. ${ }^{29}$

Recently, nanostructured materials have attracted a lot the attention for actuator design. Enhanced performances (energy efficiency, deformation rate, etc.) combined to lower triggering activation energies were observed, in particular, with surface-driven actuation mechanisms that utilize nanostructured (porous) materials, which is mainly due to the nanostructure-induced exalted surface to volume ratios. ${ }^{30-32}$ In addition to improve existing actuators technologies, such nanotailored materials allow one to benefit from physical and chemical nanoscale phenomena that could not be considered before for the mechanism of actuation. An example is the nanoporous structures that authorize liquid capillary condensation in pores with sizes between 2 and $50 \mathrm{~nm}$. Such a phenomenon has recently been suggested, and yet not investigated, as an actuation concept that would lead to humidity-induced actuation devices. It has been theorized that "hard" inorganic porous cohesive networks would have the potential to generate large mechanical forces. ${ }^{33}$ For instance, it was recently observed that liquid-gas menisci forming through capillary adsorption and desorption in mesoporous silica films are accompanied by a significant transversal compressive deformation $(\approx 1-10 \%) .{ }^{34}$ The "enormous" Laplace forces (up to few GPa) generated within the interconnected network of nanopores associated with mesoporous materials are responsible for the so-called capillary contraction. ${ }^{35,36}$ The harnessing of the Laplace force and its conversion in a macroscopic work seem to be a promising way to convert an easy to reach chemical energy into an exploitable force. A technological solution would then associate a layer of the stimulus-responsive material onto a passive elastic substrate. This is one of the simplest systems that can convert a stimulus-induced volume change of an active material into macroscopic mechanical work. ${ }^{37,38}$ The challenge here consists of elaborating the tailored nanostructured porous active layer onto the proper passive elastically deformable substrate ensuring a strong and homogeneous bonding between both materials.

In the present article, we demonstrate that such humidity-sensible actuators can easily be elaborated by applying sol-gel silica films with controlled nanoporosity on only one face of a thermally resistant polymer sheet, in analogy to the bimetallic strip designed to measure temperature. ${ }^{39}$ The thermal condensation curing, following the deposition of the silica layer, induces a contraction of the inorganic network and creates a lateral stress into the substrate that provokes the curvature of the whole sheet. Adsorption at the surface of the porous silica layer and capillary condensation in the nanopores, which are in equilibrium with the applied humidity, create elastic stresses and strains inside the whole porous materials that add to the previous stress. The result is the modification of the sheet curvature radius with respect to applied humidity. If the additional stress induces a contraction of the porous network, the curvature radius decreases, while if it induces an expansion, the radius increases, thus converting the adsorption/desorption and capillary condensation/evaporation into reversible actuation movements. We show that the evolution of curvature can be more or less complex depending on the type of porosity. The movements and forces generated by the bilayer sheet actuators were assessed through combined measurements at the nano- and macroscale by ellipsometry techniques, optical observations, and micronewton force measurements. The relatively high generated forces make this actuation concept an interesting 
potential "water-fueled" solid engine, which is illustrated by an autonomously-moving system in the following parts.

\section{Results and Discussion.}

\section{Fabrication and Characterization of Bilayer Strips.}

Bilayer structures are an assembly composed of a silica thin film and a flexible $50 \mu \mathrm{m}$ thick polyimide as substrate. Silica coatings were synthesized by classical sol-gel and dip-coating methods on polyimide (poly-4,4'-oxydiphenylenepyromellitimide) sheets from hydro-alcoholic solutions composed of TEOS (TetraEthylOrthoSilicate) as silica precursor. Mesoporous thin films were prepared using the evaporation-induced self-assembly $y^{40}$ method in the presence of block-copolymer micellar template Pluronic F127, while microporous silica xerogel films were obtained from similar solutions but free of block-copolymers. Silica thin films were deposited on both sides of a polyimide substrate by dip-coating. After being dried at room temperature when thin films are still a gel, poorly condensed with a low mechanical resistance, one face of the substrate was carefully cleaned with a tissue soaked with ethanol in order to completely remove the coating and keep a silica thin film only on one side of the substrate to get a bilayer structure. Then all films were stabilized by a thermal curing at $350{ }^{\circ} \mathrm{C}$ for $10 \mathrm{~min}$ under air (see Materials and Methods). Polyimide substrate was chosen because of its chemical inertia, thermal stability $\left(-269\right.$ and $\left.400{ }^{\circ} \mathrm{C}\right)$, and mechanical properties. ${ }^{41}$ Bilayer structures are labeled $S^{X}$ or $S^{M}$ to indicate if they were fabricated using a xerogel or mesoporous silica film, respectively.

It is known that growth of a thin film on a unique face of a substrate is susceptible to induce the substrate deformation depending on the condition of the coating formation. Considering a coating with a homogeneous thickness on a plane substrate, this deformation will be spherical and either concave when the mechanical stress created by the coating and applied on the substrate is a compressive stress $(\sigma<0)$ or convex when it is a tensile stress $(\sigma>0)$. For our silica-polyimide bilayers shown in Figure 1.a, two distinct steps are observed. First, immediately after the deposition of the silica sol-gel solution by dip-coating, solvent evaporates leading to the formation of a thin film composed of silica intermediate moieties. The drying of the sub-micrometer-thick gel film at room temperature induces total stress in the plane of the coating which is approximately equivalent to the capillary pressure in the liquid. ${ }^{42}$ Nevertheless residual stress generated by the transformation from liquid film to gel is relatively weak because the loss of thickness of the coating is accommodated by viscous and plastic strains due to the low condensation degree of the intermediate silica matrix. Therefore bilayer structures do not show any curvature after drying as shown in Figure 1.b. 

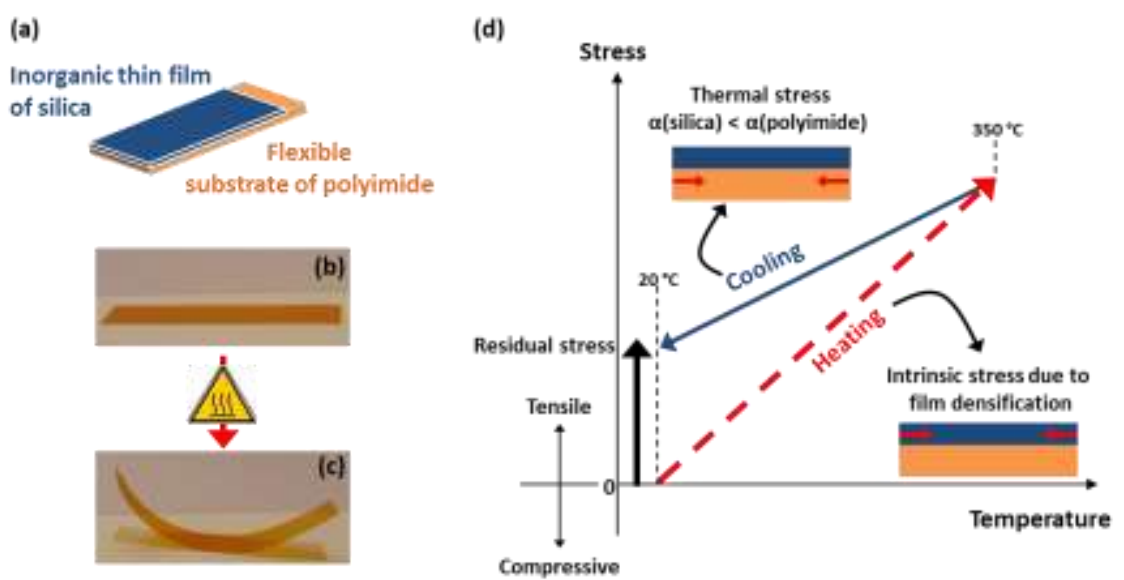

Figure 1. (a) Representation of the silica-polyimide bilayer sheet. (b) Photograph of a silica xerogel based bilayer sheet after being dried at room temperature. (c) Photograph of the corresponding bilayer sheet after calcination at $350{ }^{\circ} \mathrm{C}$ for $10 \mathrm{~min}$. (d) Schematic representation of stress evolution in silica thin films during a thermal run composed of heating and cooling steps, adapted from Ref. 44.

In the second step, the average thickness contractions of 19 and $38 \%$, measured by ellipsometry for the xerogel and mesoporous silica thin films respectively, are due to the coating densification by condensation of the inorganic network during thermal curing at $350{ }^{\circ} \mathrm{C}$ for $10 \mathrm{~min}$. Due to the thermally induced silica volume reduction and because the film is relatively strongly bonded to the polyimide substrate, the film thermal contraction is mainly accommodated by a global film volume decrease that is clearly observed through the thickness reduction. In-plane stresses, parallel to the substrate-coating interface, are then generated and make the bilayer sheets curved as shown in Figure 1.c. ${ }^{43}$ The curvature formation in such bilayer systems was explained by Kozuka et al. ${ }^{44}$ and is represented in Figure 1.d. To summarize, under heating, the condensed coatings can be considered as retained in an elongated state by the substrate, and the generated stress cannot, or very partially, be dissipated by any viscous or plastic strains. This in-plane tensile stress is called intrinsic stress (see Figure 1.d). Intensity of the intrinsic stress and resulting curvature of the sol-gel/polyimide bilayers depend on thermal curing conditions, crystalline or amorphous nature of the coating and on the hydrolysis ratio of the inorganic solution. ${ }^{45-47}$ No significant chemical reaction is expected to occur during cooling to room temperature $\left(20^{\circ} \mathrm{C}\right)$. Nevertheless the drop in temperature is accompanied by a decrease of the bilayer curvature. Indeed thermal stress is generated due to the difference of thermal expansion coefficients $(\alpha)$ between silica and the polyimide substrate. The lower $\alpha$ value of silica compared to that of polyimide, $\alpha_{\mathrm{si}}=5.5 \times 10^{-7}{ }^{\circ} \mathrm{C}^{-1}$ and $\alpha_{\mathrm{K}}=30-60 \times 10^{-6}{ }^{\circ} \mathrm{C}^{-1}$ respectively, induces a compressive stress opposed to the tensile intrinsic stress. This thermal expansion coefficient related stress is negligible during the heating step. ${ }^{48}$ As a result, the curvature observed at room temperature corresponds to the residual stress, which is the sum of intrinsic and thermal stresses.

For the sake of simplicity, a rectangular flat geometry has been chosen for the bilayers fabrication $\left(L \times I=60 \times 6 \mathrm{~mm}^{2}\right)$. This geometry disfavors the transversal (I direction) in-plane stress and thus reduces the formation of a curvature along the width of the sample. From now on, only longitudinal ( $L$ direction) in-plane stress will be considered. At least $50 \mathrm{~nm}$ of coating was required to bend the substrate (see Figure 2.a). The residual in-plane stress in bilayer structures can be estimated by measuring the curvature radius of the elastic isotropic substrate where the coating is deposited using Stoney's equation (Eq. 1): 


$$
\sigma_{f}=\frac{E_{s} h^{2}}{6 t_{f}\left(1-v_{s}\right) R} \quad \text { Eq. } 1
$$

where $\sigma_{f,} E_{s}, h, t_{f}, v_{s}$, and $R$ are the residual stress in the film, the Young's modulus of the substrate $\left(E_{s}=2.5 \mathrm{GPa}\right.$ at $25^{\circ} \mathrm{C}$ ), the thickness of the substrate, the thickness of the silica coating, Poisson's coefficient of the substrate $\left(v_{s}=0.3\right)$ and the radius of curvature of the bilayer sheet respectively. ${ }^{49,50}$
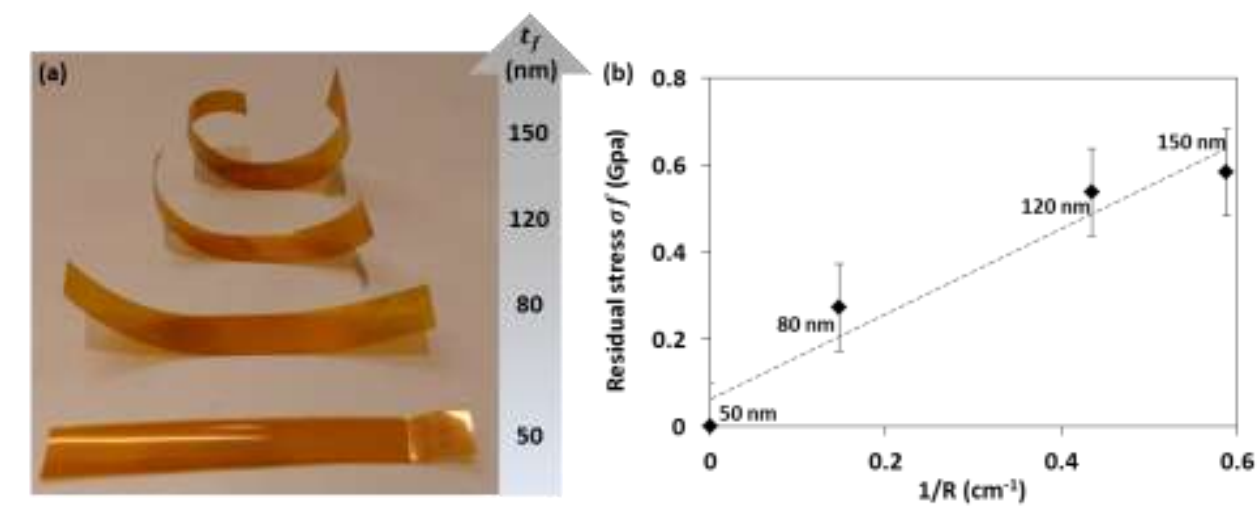

Figure 2. (a) Photograph of the curvature of $50 \mu \mathrm{m}$-thick polyimide strips combined with a layer of xerogel silica with decreasing thickness from top to bottom. (b) Graphic of the corresponding calculated residual stress deduced from Eq. 1.

The residual stress determined by Stoney's equation increases linearly with the xerogel coating thickness, as reported in Figure 2.b. A residual stress of around $500 \mathrm{MPa}$ was determined for the thicker silica coating $(150 \mathrm{~nm})$. In-plane stress values of the same order were determined by Parrill et al. ${ }^{46} \mathrm{~S}^{\mathrm{X}}$ and $\mathrm{S}^{\mathrm{M}}$ bilayer strips, showing radii of curvature $(R)$ of $5.0 \pm 0.3$ and $6.8 \pm 0.3 \mathrm{~cm}$ at $10 \%$ of relative humidity, respectively, were obtained with $80 \pm 5$ and $252 \pm 5 \mathrm{~nm}$ thick xerogel and mesoporous silica thin films, respectively. The higher curvature of $S^{\mathrm{X}}$ compared to that of $\mathrm{S}^{\mathrm{M}}$ despite a significantly lower thickness reveals that, in addition to the thin film thickness, the pore network structure also influences directly the residual stress. This highlights the fact that in-plane residual stress is related to the density of the porous material and to the pore characteristics. The smaller the size of the pores and the higher the siliceous network density, the higher the stress generated during thermal curing.

Porosity and pore size distribution of both silica films were assessed by environmental ellipsometry porosimetry. Corresponding water adsorption-desorption isotherms are reported in Figure 3.a and 3.b for both silica films deposited on plane silicon (100) substrates, assuming the structures are the same as those on polyimide substrates. The isotherm of the xerogel film shows a water-uptake mainly for relative humidity below $20 \%$, which is characteristic of microporosity. Such a xerogel typically contains $<2 \mathrm{~nm}$ diameter micropores, because the curing temperature of $350{ }^{\circ} \mathrm{C}$ is not high enough to completely densify the inorganic network. The water adsorption/desorption within the mesoporous silica film shows the expected typical type IV isotherm of an interconnected pore network (Figure 3.b). Pore volumes of 10 and $49 \%$ were determined for xerogel and mesoporous films, respectively, using the Bruggeman effective medium approximation. ${ }^{51}$ Capillary condensation is observed at water partial vapor pressure of 0.70 for the mesoporous film. The pore size distribution was determined using the Kelvin equation (Eq. 2) for mesoscopic pores: 


$$
\ln \frac{P}{P_{0}}=-\frac{2 \gamma V_{1} \cos \theta}{r_{\mathrm{p}} R T} \quad \text { Eq. } 2
$$

where $P / P_{0}$ is the relative pressure and $r_{\mathrm{p}}, \gamma, V_{1}, \theta, R$, and $T$ are the Kelvin pore radius, the surface tension, the molar volume of liquid, the contact angle, the gas constant and the temperature, respectively.

Pore size distribution taken from adsorption curve (Figure 3.b - inset) shows Gaussian-like distribution in the range of 4.3-8.1 nm, with a corresponding mean pore diameter of $5.5 \pm 0.40 \mathrm{~nm}$. Because liquid water / vapor interfaces are suspected not to exist below $2 \mathrm{~nm}$, the Kelvin equation is not adapted to micropores of the xerogel film. We thus consider that xerogel films contain micropores below $2 \mathrm{~nm}$ in diameter, with a large size distribution. GI-SAXS pattern of the mesoporous silica film is shown in Figure 3.b (inset), it corresponds to a typical pseudo-cubic (Im3m) structure. ${ }^{52}$ 

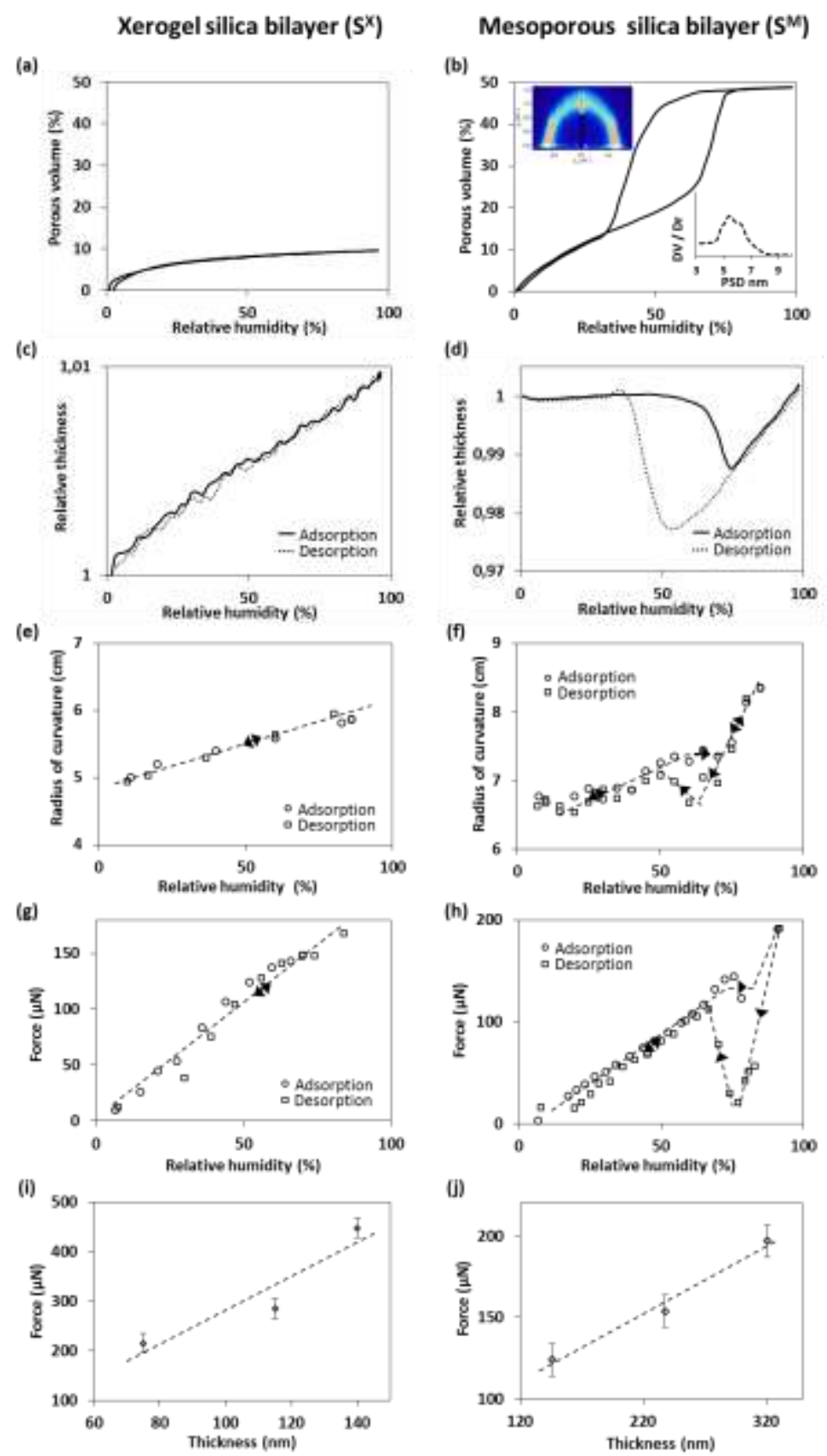

Figure 3. (a,b) Water adsorption-desorption isotherms of the microporous xerogel and the mesoporous F127-templated silica thin films on silicon substrates, with the corresponding pore size distribution, and the GISAXS pattern (inset) for the mesoporous thin film. (c,d) Relative thickness evolution depending on relative humidity at room temperature for microporous xerogel and mesoporous F127-templated silica thin films on silicon substrates. $(e, f)$ Radius of curvatures of microporous xerogel $\left(S^{X}\right)$ and mesoporous $\left(S^{M}\right)$ silica-polyimide bilayer strips depending on relative humidity at room temperature. $(\mathrm{g}, \mathrm{h})$ Force (measured by sensor compression) generated by $\mathrm{S}^{\mathrm{x}}$ and $S^{M}$ actuators depending on relative humidity at room temperature. $(1, j)$ Values of force for microporous $\left(S^{X}\right)$ and mesoporous $\left(S^{M}\right)$ actuators versus the thickness of silica layers at $R H=90 \%$. 


\section{From Bilayer Strips to Actuator.}

Actuation works of the silica-polyimide bilayer strips were investigated by fixing $\mathrm{S}^{\mathrm{X}}$ or $\mathrm{S}^{\mathrm{M}}$ samples in an environmental chamber with controlled atmosphere at room temperature (see Figure 4.a). Humidity in the chamber was controlled between $10 \%$ and $85 \%$ using a RegulHum flow system from SolGelWay Company. A low flow of $1 \mathrm{~L}$. $\mathrm{min}^{-1}$ was used to avoid an oscillating movement of the strips. The photos displayed in Figure 4.b show the variations of the strip curvature with respect to the applied humidity for both samples, confirming the efficient conversion of humidity stimulus into macroscopic mechanical movement for the two types of porous structures.
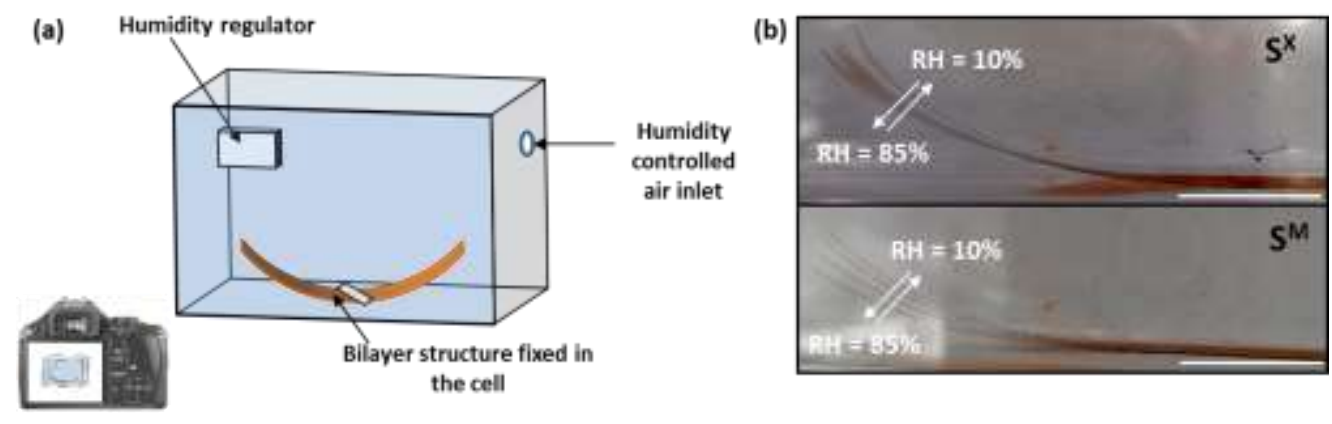

Figure 4. (a) Representation of the setup used for the dynamic in situ investigation of the curvature radius depending on the humidity for $\mathrm{S}^{\mathrm{X}}$ and $\mathrm{S}^{\mathrm{M}}$ bilayer strips. (b) Photos of $\mathrm{S}^{\mathrm{X}}$ and $\mathrm{S}^{\mathrm{M}}$ bilayers, depending on the relative humidity between $10 \%$ and $85 \%$ at room temperature. Scale bar corresponds to $1 \mathrm{~cm}$.

\section{Response of Xerogel Microporous Thin Films:}

To understand the actuation mechanism, the effect of water adsorbed onto the top surface and in the micropores of the silica xerogel has been observed in situ by recording the variations of the curvature of the $S^{x}$ bilayers with the relative humidity in the chamber. Results are presented in Figure 3.e. One observes that the increase in humidity is accompanied by a linear augmentation of the curvature radius from $R\left(\mathrm{~S}^{\mathrm{x}}\right)=4.9 \pm 0.3 \mathrm{~cm}$ for $\mathrm{RH}=9.5 \%$ to $R\left(\mathrm{~S}^{\mathrm{x}}\right)=5.8 \pm 0.3 \mathrm{~cm}$ for $\mathrm{RH}=86 \%$. This suggests, according to Stoney's theory, that stress is generated by water adsorption into the microporous silica-polyimide bilayer structure. Because the polyimide substrate has a low moisture sensitivity, ${ }^{53}$ variation of curvature originates from the microporous silica part. Decrease of the bilayer curvature from low to high RH indicates that humidity induces a compressive stress, which is fully reversible upon adsorption/desorption cycles.

Samuel et $a .^{54}$ reported similar monotonic compressive stress for microporous silica thin films deposited on a silicon wafer and exposed to the vapor of wetting liquid (methanol, ethanol, etc.) They showed that such compressive stress results from changes in the solvent-induced stresses (solvation stresses) in the porous network. Applying DFT model, the magnitude of such compressive solvation stress for a microporous coating was demonstrated to be sensitive to the pore size and the degree of polydispersity in size for a given sorbate molecule. ${ }^{54}$ In the case of a humid atmosphere, sorption of water molecules in microporous silica xerogel probably starts for very low humidity rates $^{54}$ (lower than the probed range of 9.5 to $86 \%$ ) and is completed for $\mathrm{RH}<20 \%$, as shown in Figure 3.a. However, the compressive stress is still increasing at high relative vapor pressure (up to measured $\mathrm{RH}=86 \%$ ), highlighting that solvation stress still increases even when micropores are filled with a "liquid-like" condensed fluid. 
Evolution of the thickness induced by water adsorption and desorption in xerogel silica films deposited on silicon was assessed by in situ environmental ellipsometry and is reported in Figure 3.c. Here, again, a reversible linear film transversal swelling is observed during the cycle of controlled humidity starting from 2 to $98 \%$, without the presence of capillary contraction, confirming the lack of mesopores. The out-of-plane reversible strain of $1 \%$ demonstrates the adsorption-induced deformation of the nanoporous silica xerogel coating. Similar behaviors of the curvature and film thickness with relative humidity show that solvation stress is isotropic in the microporous silica coating. Indeed, investigation of curvature radius provides direct information on longitudinal in-plane stress whereas environmental ellipsometry detailsstress perpendicular to the silica-substrate interface.

Adsorption-induced deformation of microporous materials was first described by Bangham et al. and Meehan on coal and is usually called Bangham's effect. ${ }^{55,56}$ Recent work linked such deformations with the solvation stress exerted on the micropore walls. ${ }^{57}$ Whereas positive solvation stress can be explained by high fluid densities inside the micropores for which short range repulsive interactions between fluid-fluid and fluid-solid molecules become preponderant, negative solvation stress results from loose molecular packing. ${ }^{58}$

It is therefore reasonable to think that water molecules in micropores of the silica xerogel are already highly packed at $\mathrm{RH}=9.5 \%$ at the beginning of the experiments, forming a "liquid-like" condensed fluid as illustrated in Figure 5.a. By increasing the relative humidity, no more significant water sorption inside pores is expected. Nevertheless, positive solvation stress continues to increase due to short-range repulsive interactions that increase along with the water molecule packing, which grows with the surrounding water vapor pressure augmentation. Positive solvation stress induces swelling of pore volume that we estimate about $1 \%$ according to the out-of-plane reversible strain measured by environmental ellipsometric porosimetry (EEP), see Figure 3.c, assuming the isotropicity of the solvation stress and the insensitivity to water adsorption of the residual stress contained in the solid network of the porous material. Such relatively high deformation value of pore size ${ }^{54}$ can be imputed to the relative low annealing temperature $\left(T=350^{\circ} \mathrm{C}\right)$ of the silica thin films, which enhanced the silica network flexibility by reducing, slightly, the siloxane bond density. The much better flexibility of the polyimide substrate compared to that of the usual silicon substrate also drastically reduces the mechanical constraint applied to the silica matrix, which favors a large porous network breathing. The result is the creation of compressive stress into the bilayer actuator which is responsible for a significant reduction of the strip's initial curvature with a centimeter scale deflection (see Figure 4.b). During desorption, the inverse process occurs, leading to the decrease of the solvation forces without noticeable hysteresis and thus the return to the initial curvature.

\section{Response of the F127-Templated Mesoporous Thin Film:}

Influence of water adsorption and desorption for the mesoporous-responsive silica thin film on a silicon substrate and for the $\mathrm{S}^{\mathrm{M}}$ bilayer strips has been studied with the same approach used for the previous xerogel systems. Results are shown in Figure 3.d and 3.f. The response of the mesoporous film is radically different than with the xerogel one. A multistep increase of the curvature radius is observed upon humidity increase with a first linear section from $R\left(S^{\mathrm{M}}\right)=6.5 \pm 0.3 \mathrm{~cm}$ to $R\left(\mathrm{~S}^{\mathrm{M}}\right)=7.3 \pm$ $0.3 \mathrm{~cm}$ between $\mathrm{RH}=15 \%$ and $\mathrm{RH}=55 \%$. It is followed by a second section with a stable value of $R\left(\mathrm{~S}^{\mathrm{M}}\right)=7.3 \pm 0.3 \mathrm{~cm}$ between $\mathrm{RH}=55 \%$ and $\mathrm{RH}=72 \%$. Finally, the radius of curvature increases from $R\left(\mathrm{~S}^{\mathrm{M}}\right)=7.3 \pm 0.3 \mathrm{~cm}$ to $R\left(\mathrm{~S}^{\mathrm{M}}\right)=8.3 \pm 0.3 \mathrm{~cm}$ between $\mathrm{RH}=72 \%$ and $\mathrm{RH}=85 \%$. Desorption is characterized by three steps, too. First, $R\left(\mathrm{~S}^{\mathrm{M}}\right)$ changes from $8.3 \pm 0.3 \mathrm{~cm}$ to $R\left(\mathrm{~S}^{\mathrm{M}}\right)=6.6 \pm 0.3 \mathrm{~cm}$ 
between $\mathrm{RH}=85 \%$ and $\mathrm{RH}=62 \% . R\left(\mathrm{~S}^{\mathrm{M}}\right)$ increases back to $7.1 \pm 0.3 \mathrm{~cm}$ down to $\mathrm{RH}=50 \%$. Between $\mathrm{RH}=50 \%$ and $\mathrm{RH}=15 \%, R\left(\mathrm{~S}^{\mathrm{M}}\right)$ evolves from $7.1 \pm 0.3 \mathrm{~cm}$ to $R\left(\mathrm{~S}^{\mathrm{M}}\right)=6.5 \pm 0.3 \mathrm{~cm}$. Similar to microporous silica film, the mesoporous bilayer actuation is powered by the water affinity with silica. However, the whole behavior of the mesoporous film shows a hysteresis loop, in contrast to the xerogel film.

Similarly, $S^{M}$ thickness evolution in Figure $3 . d$ shows a hysteresis loop with two distinct capillary contractions due to the capillary condensation and the capillary desorption of water into the pores, confirming the presence of mesopores. On the other hand, no significant thickness variation is observed at low pressures, suggesting that the Bangham's effect observed with the microporous thin films is hidden by the larger volume of mesopores, which are responsible for the dominant capillary contractions. It is likely that positive solvation stress due to water adsorption in the micropores, which are also present in the mesoporous inorganic network, takes place with an effect similar to that of the one observed for the microporous xerogel bilayer..$^{59}$ It produces a compressive stress, resulting in the increase of the radius of curvature, as confirmed in Figure 3.f. Contrary to xerogel films, no transversal swelling is observed, which is probably due to the presence of mesopores that makes the network more flexible for isotropic stress relaxation (see Figure 5.b. step II). Indeed, the mesopore network is probably flexible enough to be able to accommodate the perpendicular strain due to micropores' "dilatation" induced by the adsorption, resulting in the "masking" of the transversal adsorption-induced deformation usually observed for pure microporous materials.

Between $55 \% \leq \mathrm{RH} \leq 75 \%$, we observed the contraction of the mesoporous silica film on silicon substrates, whereas the radius of curvature $R\left(\mathrm{~S}^{\mathrm{M}}\right)$ keeps constant for silica-polyimide bilayer strips. This plateau of curvature seems to result from the balance between the compressive stress induced by the positive solvation forces in micropores and the tensile stress developed during the capillary contraction of the mesopores created by capillary condensation. In this case the stability of the curvature (from $\mathrm{RH}=55 \%$ to $\mathrm{RH}=72 \%$ ) suggests that compression and tension have similar intensities.

With $\mathrm{RH} \geq 72 \%$ both film thickness on silicon substrates and radius of curvature of polyimide bilayers significantly increase, illustrating the generation of large compressive stress in a mesoporous-polyimide bilayer. The higher slope of the curvature radius versus humidity compared to that observed for $15 \% \leq \mathrm{RH} \leq 55 \%$ can probably be imputed to the stiffening of the silica layer due to the water filling of mesopores that prevents mesopores from dissipating at high humidity, a part of the compressive stress generated by micropores and the related adsorption-induced deformation of the thin film, as illustrated in Figure 5.b. step III.

During desorption, values of $R\left(\mathrm{~S}^{\mathrm{M}}\right)$ do not take the same return path for the intermediate humidity $(50 \% \leq \mathrm{RH} \leq 72 \%)$ due to the shift in vapor pressure, at which desorption and adsorption take place (see figure 5.b step IV). The local minimal value of $R\left(S^{\mathrm{M}}\right)=6.6 \pm 0.3 \mathrm{~cm}$ is associated with a breathing movement of the actuator, which comes from two combined phenomena, inducing both an abrupt contraction of the silica layer: (i) the intense tensile stress generated by the isotropic capillary contraction and (ii) the decrease of the compressive stress induced by the lower water molecule packing at desorption. For $\mathrm{RH}<50 \%$, the bilayer strip recovers slowly its initial curvature through the step-by-step decrease of the compressive stress accompanying the diminution of solvation stresses with the decrease of humidity. 


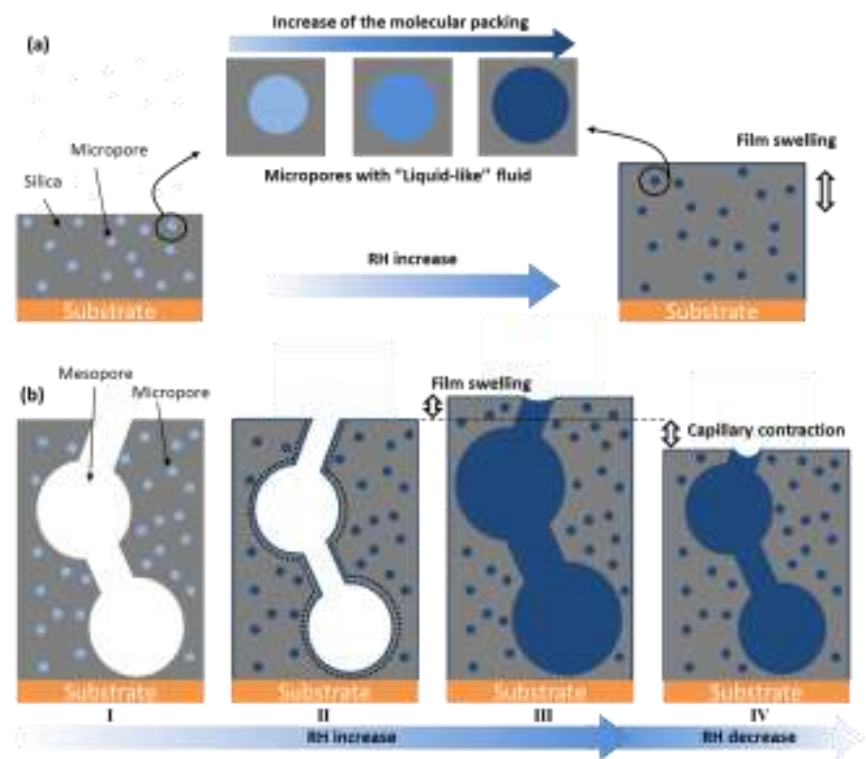

Figure 5. Scheme of the dynamic water-adsorbed actuation mechanisms occurring in (a) microporous- and (b) mesoporous-responsive silica thin film for $\mathrm{S}^{\mathrm{X}}$ and $\mathrm{S}^{\mathrm{M}}$ actuators.

According to Stoney's equation, in-plane stress modulation of 15 and $19 \%$ was obtained for $\mathrm{S}^{\mathrm{x}}$ and $\mathrm{S}^{\mathrm{M}}$ actuators, respectively, when passing from $\mathrm{RH}=10 \%$ to $\mathrm{RH}=85 \%$. It is clear from the latter results that $\mathrm{RH}$ can be used to actuate systems in combination with nanoporous inorganic thin films prepared by sol-gel techniques. Increasing the porous volume as well as the fine control of the pore size distribution ${ }^{54}$ is probably the easiest way to enhance the mechanical movement of the silicapolyimide actuators. The resulting increase of the surface of contact between water and silica should favor generation of a higher solvation force in whole microporous films and a higher network deformation during capillary condensation/desorption for mesoporous films.

\section{Evaluation and Tuning of Actuation Force and Speed:}

In this part, we evaluate the performance of the hybrid silica-polyimide actuators presented above. The effects of pore size and thickness of the responsive silica coatings on the force generated by the bilayer actuators have been deduced from in situ measurements of the force applied on a microforce sensing probe induced by the variation of the relative humidity in a sealed chamber (see Figure 6) and are reported in Figures 3.g and 3.h. As expected, the force globally builds up with the increase of humidity, similar to the variation of radius of curvature presented in Figure 3.e and 3.f. This shows that compressive and tensile stresses generated by water adsorption and capillary condensation can be converted into a usable, reversible, anisotropic force through a simple bilayer strip actuator. 


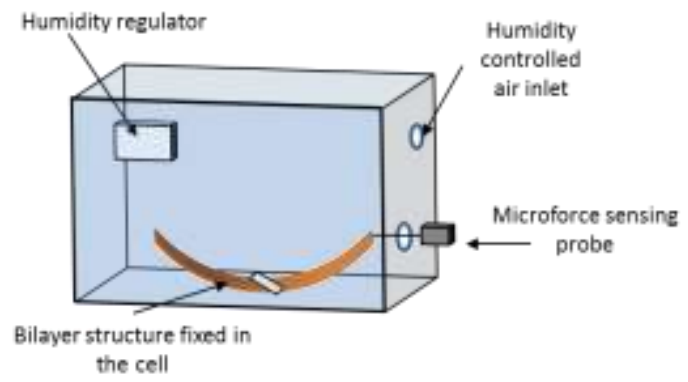

Figure 6. Representation of the setup used for the dynamic in situ investigation of the forces generated by the $S^{\mathrm{X}}$ and $\mathrm{S}^{\mathrm{M}}$ bilayer actuators induced by the variation of relative humidity.

Forces up to $450 \mu \mathrm{N}$ could be generated with the previous device composition and dimension. Like the radius of curvature, the xerogel gives a fully reversible linear tendency, while the mesoporous film has a more complex behavior with a linear tendency at low pressures accompanied by a hysteresis loop at high pressure that one can logically attribute to capillary condensation. The shift of minimal hysteresis values for the radius of curvature (Figure 3.f) compared to the measured force (Figure 3.h) does not have an evident rational explanation. It could, however, be attributed to a variation of temperature between the atmospheres of saturated vapor generation and of the measurement chamber. These very different force profiles indicate that this actuation can be used for either a progressive or a sudden conversion of energy into force, depending on the pore size. The force can also be adjusted through the tuning of the porous film thickness, in which the stresses (tensile or compressive) are generated. The forces developed for a relative humidity of $90 \%$ for various thicknesses are reported in Figure 3.i and 3.j for both types of coatings combined with a 50 $\mu \mathrm{m}$ thick polyimide. As expected, the developed forces increase quasi-linearly, with the thickness of the sensitive coating reaching $450 \pm 20$ and $200 \pm 10 \mu \mathrm{N}$ for a $140 \pm 5 \mathrm{~nm}$ thick xerogel coating and for a $320 \pm 5 \mathrm{~nm}$ thick mesoporous coating, respectively.

The linear augmentation indicates that solvation forces increase with thickness and their effects on the global compressive stress in the bilayer are the same regardless of the localization of their original micropores in the silica coating. It is worth noticing that values measured for the mesoporous actuators are significantly weaker than that for the xerogel, despite the lower thickness and porosity of the latter. In other words, greater forces would likely be created by increasing the ratio of microporous volume to mesoporous volume and thickness of the layer.

Concerning the role of the polyimide substrate, force values of $910 \pm 40$ and $450 \pm 20 \mu \mathrm{N}$ were recorded at $\mathrm{RH}=90 \%$ for bilayer strips composed by a $140 \mathrm{~nm}$ thick xerogel layer deposited on a 75 $\mu \mathrm{m}$ thick and a $50 \mu \mathrm{m}$ thick polyimide substrate, respectively. A thicker polyimide layer allowed development of a higher force through water adsorption due to a higher rigidity and also to its contribution on the stocked residual stress in the coating after thermal treatment (see Figure 2). In return, a lower movement of amplitude was obtained for the thicker polyimide, confirming the classical compromise between the generated force and the amplitude of movement.

In addition, a complete characterization of an actuator requires the study of actuation speed. A fast actuation speed is crucial to consider the integration of the actuator in a device. In Table 1, the actuator deflection speeds in radius of curvature are reported, depending on relative humidity for xerogel $\left(S^{x}\right)$ and mesoporous $\left(S^{M}\right)$ silica films obtained from Figure 3.e and 3.f. A xerogel silica actuator, as seen previously, shows a reversible linear increase of the curvature radius with a constant speed of $1 \% . \%^{-1}$ (percentage of deformation every one percent of $\mathrm{RH}$ ) on all ranges of 
humidity. A similar magnitude of speed (i.e. $0.6 \% . \%^{-1}$ ) for $\mathrm{S}^{\mathrm{M}}$ is observed for low pressures before capillary condensation. After capillary condensation, a reversible linear increase of the curvature radius is observed with a much higher speed value of $4 \% . \%^{-1}$. The sudden increase of the humidity response of mesoporous actuators is similar to the voltage threshold for electronic diodes. Such mechanical actuators, showing an abrupt response with humidity threshold that is tunable through the size of pores, could be exploited as a mechanical circuit breaker or switch.

Table 1. Relative speed of the radius of curvature variation versus relative humidity depending on the range of relative humidity for $\mathrm{S}^{\mathrm{X}}$ and $\mathrm{S}^{\mathrm{M}}$ actuators.

\begin{tabular}{ccc} 
& $\mathrm{S}^{\mathrm{X}}$ & $\mathrm{S}^{\mathrm{M}}$ \\
\hline Speed 1 $\left(\% . \%^{-1}\right)$ & $1(9,5 \% \leq \mathrm{RH} \leq 86 \%)$ & $0.6(7 \% \leq \mathrm{RH} \leq 50 \%)$ \\
Speed 2(\%.\% $\left.{ }^{-1}\right)$ & - & $4(70 \% \leq \mathrm{RH} \leq 85 \%)$ \\
\hline
\end{tabular}

We demonstrated that reversible compressive stress and capillary contraction inducing the movement of actuators were triggered by water molecule adsorption in the pores of silica layers (mesopores, micropores). A second investigation of the actuation speed has been performed by measuring in parallel the force (using a microforce probe) and the relative humidity (using a lab hygrometer) as a function of time. The mechanical response always stabilized faster than the hygrometric one, suggesting that, even if limited by the diffusion of gaseous water for both silica coating and hygrometer-sensitive surface, the actuator shows a quasi-instantaneous response.

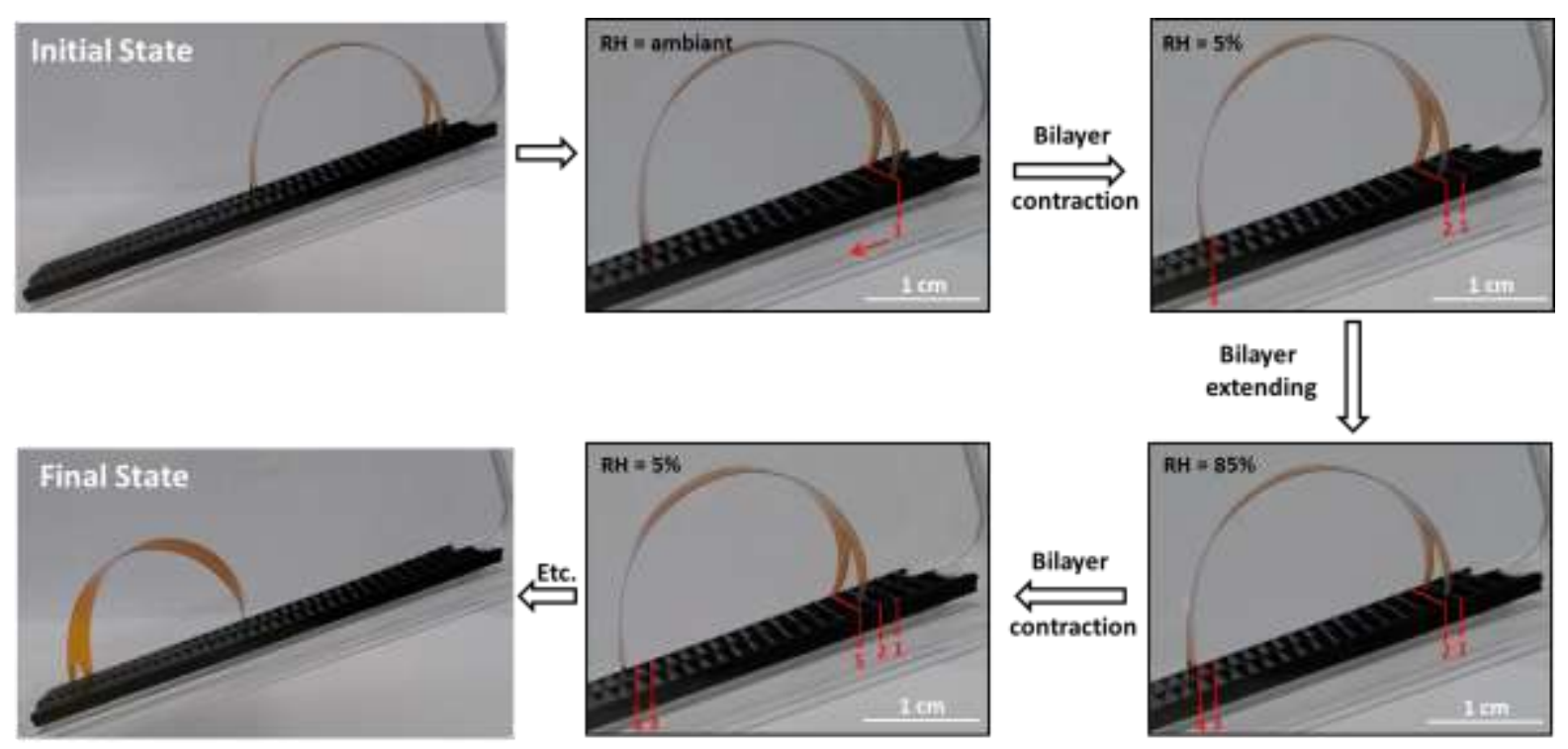

Figure 7. Illustration of the step-by-step movement of a bilayer actuator ( $50 \mu \mathrm{m}$ thick polyimide layer combined with a $110 \pm 5 \mathrm{~nm}$ xerogel silica film) placed on a tilted $\left(\approx 15^{\circ}\right)$ rack and pinion in humiditycontrolled chamber for humidity rate oscillations between $5 \%$ and $85 \%$.

The Walking Strip: Example of RH-Stimulated Actuation.

A bilayer actuator, optimized for large movement amplitude combined with high generated force of contraction and extension, has been obtained with a $110 \pm 5 \mathrm{~nm}$ xerogel silica film deposited on a $50 \mu \mathrm{m}$ thick polyimide layer. The curved actuator can stay standing-up on its four "legs" due to its relatively high rigidity but can perform a back and forth curvature variation under oscillating humidity. The actuator direction of displacement was directed by the use of a simple tilted rack with 
slightly inclined steps (see Figure 7). When a dry atmosphere is applied in the chamber, the actuator contracts inducing the jump of the upper legs from position 1 to position 2. Increasing the humidity induces the actuator extension and the jump of the lower legs from position 3 to position 4 , with the upper legs being blocked by the step. The repeated contractions and expansions of the actuator drive it down to the end of the rack (see the movie available in the Supporting Information), and confirm the relatively good stability of pure silica porous coatings over several repeated humidity cyclings. ${ }^{35}$

\section{Conclusions.}

It was shown that capillary condensation and/or water molecule adsorption in mesoporous and xerogel-based silica thin films could be converted into mechanical force using actuators with a bilayer architecture to amplify the water-induced nanostrain of a thin silica film backed on a thermosresistant polyimide layer and provide usable mechanical work at the macroscopic scale. We demonstrate that reversible solvation stress due to water sorption in micropores and Laplace forces induced during capillary condensation in mesopores were responsible for the fast bilayer strip actuation. In view of the different responses obtained in this study, it is clear that one would be able to finely tune the mechanical response (speed, amplitude of movement, degree of force, force $v s \mathrm{RH}$ profile) by using not only the pore size distribution, but also the porous volume, the morphology, and orientation of the pores, the interconnectivity between them, the combination of various types of pores, without forgetting the composition, the structure of the matrix itself, as well as the substrate interface and mechanical properties. This fundamental work provides insight into surface-chemistrydriven actuation systems implying hard ceramic as responsive materials. Such strategy has the potential to be used in future for energy harvesting devices or chemically driven muscles for robotics.

\section{Materials and Methods.}

Chemical reactants:

Absolute ethanol 100\% (EtOH), TEOS (TetraEthylOrthoSilicate), hydrochloride acid 37\%, F127 Pluronic $\left(\mathrm{EO}_{106} \mathrm{PO}_{70} \mathrm{EO}_{106}\right)$, were purchased from Aldrich. DuPont ${ }^{\mathrm{TM}}$ Kapton $^{\circledR} \mathrm{HN}$ sheets (polyimide) were purchased from Radiospares. All products were used as received.

Film processing:

Xerogel silica thin films, samples labeled $S^{x}$, were prepared from solutions composed of TEOS/ $/ \mathrm{HCl} / \mathrm{H}_{2} \mathrm{O} / \mathrm{EtOH}$ with respective molar ratios of 1:0.15:4.4:38. Mesoporous silica thin films with F127 template (labeled $\mathrm{S}^{\mathrm{M}}$ ) were prepared from solutions composed of TEOS/F127/ $/ \mathrm{HCl} / \mathrm{H}_{2} \mathrm{O} / \mathrm{EtOH}$ with respective molar ratios of 1:0.004:0.005:5:41.

TEOS was first dissolved in $\mathrm{EtOH}, \mathrm{HCl}(2 \mathrm{M})$ and $\mathrm{H}_{2} \mathrm{O}$ before potential addition of the template. Solutions were stirred at least for $24 \mathrm{~h}$ at room temperature before use. Films were prepared both on silicon wafer and $50 \mu \mathrm{m}$-thick Kapton substrate by dip-coating at room temperature and relative humidity of $10 \pm 5 \%$ with constant withdrawal rates comprised between 3.7 and $12 \mathrm{~mm} . \mathrm{s}^{-1}$. After coating on both sides of Kapton, one face of the substrate was carefully cleaned with a tissue soaked with ethanol in order to remove the coating and keep a silica thin film on one side only. Then a 60 $\mathrm{mm}$ long and $6 \mathrm{~mm}$ large strips were cut and immediately calcined at $350^{\circ} \mathrm{C}$ for $10 \mathrm{~min}$.

\section{Characterization:}


Spectroscopic ellipsometry and Environmental Ellipsometric Porosimetry (EEP) analyses were performed on a UV-visible variable angle spectroscopic ellipsometer (M2000 Woolam) equipped with a controlled atmosphere cell, in which relative vapor pressure of $\mathrm{H}_{2} \mathrm{O}$ was adjusted using mass flow controllers at room temperature $\left(20 \pm 2{ }^{\circ} \mathrm{C}\right) .{ }^{35} \mathrm{Films}$ thicknesses and refractive index were extracted from conventional $\Psi$ and $\Delta$ dispersions using a Cauchy model (Wvase 32 software).

For grazing incident small-angle X-ray scattering (GI-SAXS) a Rigaku S-max 3000 apparatus equipped with a microfocus source $\lambda=0.154 \mathrm{~nm}$. The angle of incidence was $0.2^{\circ}$.

Curvature radii $(R)$ were determined using ImageJ software from pictures of samples placed in a controlled atmosphere cell in which relative humidity was controlled by mass flow controllers.

Microforce measurements were performed using a FemtoTools FT-S microforce sensing probe. Sample were fixed in humidity controlled chamber, the sensor was placed in contact with the bended end of the sample in perpendicular position. Compression force was measured between $50 \mathrm{nN}$ and $1000 \mu \mathrm{N}$ at $20 \mathrm{~Hz}$ depending on the relative humidity.

\section{ASSOCIATED CONTENT}

Supporting Information.

Supporting Information is available free of charge on the ACS Publications website : http://pubs.acs.org.

$\mathrm{SI}$ : Video of the step-by-step moving of a bilayer actuator in controlled atmosphere.

\section{AUTHOR INFORMATION}

\section{Corresponding Author}

*E-mail: mickael.boudot@gmail.com.

\section{ACKNOWLEDGMENT}

M. Boudot et al. wants to sincerely thank Dr. Cédric Boissière, Dr. Marco Faustini and Dr. Arnaud Antkowiak for their fruitful discussion and experimental help as well as Mohamed Selmane for GISAXS experiments. We are grateful for funding provided by CIFRE fund granted by ANRT and the AVATAR project which is supported by Polyrise SAS, and DGA.

\section{References.}

(1) Crawley, E. F.; De Luis, J. Use of Piezoelectric Actuators as Elements of Intelligent Structures. AIAA J. 1987, 25, 1373-1385.

(2) Yu, Y.; Nakano, M.; Ikeda, T. Photomechanics: Directed Bending of a Polymer Film by Light. Nature 2003, 425, 145.

(3) Osada, Y.; Okuzaki, H.; Hori, H. A Polymer Gel with Electrically Driven Motility. Nature 1992, $355,242-244$.

(4) Jager, E. W.; Smela, E.; Inganäs, O. Microfabricating Conjugated Polymer Actuators. Science 2000, 290, 1540-1545.

(5) Li, Q.; Liu, C.; Lin, Y.; Liu, L.; Jiang, K.; Fan, S. Large-Strain, Multiform Movements from Designable Electrothermal Actuators Based on Large Highly Anisotropic Carbon Nanotube Sheets. ACS Nano 2015.

(6) Baughman, R. H. Carbon Nanotube Actuators. Science (80-. ). 1999, 284, 1340-1344. 
(7) Saito, Y.; Takao, H.; Tani, T.; Nonoyama, T.; Takatori, K.; Homma, T.; Nagaya, T.; Nakamura, M. Lead-Free Piezoceramics. Nature 2004, 432, 84-87.

(8) Park, S.-E.; Shrout, T. R. Ultrahigh Strain and Piezoelectric Behavior in Relaxor Based Ferroelectric Single Crystals. J. Appl. Phys. 1997, 82, 1804.

(9) Detsi, E.; Punzhin, S.; Rao, J.; Onck, P. R.; De Hosson, J. T. M. Enhanced Strain in Functional Nanoporous Gold with a Dual Microscopic Length Scale Structure. ACS Nano 2012, 6, 3734 3744.

(10) Jin, H. J.; Wang, X. L.; Parida, S.; Wang, K.; Seo, M.; Weissmüller, J. Nanoporous Au-Pt Alloys as Large Strain Electrochemical Actuators. Nano Lett. 2010, 10, 187-194.

(11) Behl, M.; Lendlein, A. Shape-Memory Polymers. Mater. Today 2007, 10, 20-28.

(12) Lai, A.; Du, Z.; Gan, C. L.; Schuh, C. a. Shape Memory and Superelastic Ceramics at Small Scales. Science (80-. ). 2013, 341, 1505-1508.

(13) Schurch, K. E.; Ashbee, K. H. G. A near Perfect Shape-Memory Ceramic Material. Nature, 1977, 266, 706-707.

(14) Krulevitch, P.; Lee, A. P.; Ramsey, P. B.; Trevino, J. C.; Hamilton, J.; Northrup, M. A. Thin Film Shape Memory Alloy Microactuators. J. Microelectromechanical Syst. 1996, 5, 270-282.

(15) Lendlein, A.; Jiang, H.; Jünger, O.; Langer, R. Light-Induced Shape-Memory Polymers. Nature 2005, 434, 695-697.

(16) Kim, J.; Chung, S. E.; Choi, S.-E.; Lee, H.; Kim, J.; Kwon, S. Programming Magnetic Anisotropy in Polymeric Microactuators. Nat. Mater. 2011, 10, 747-752.

(17) Fratzl, P.; Barth, F. G. Biomaterial Systems for Mechanosensing and Actuation. Nature 2009, 462, 442-448.

(18) Elbaum, R.; Zaltzman, L.; Burgert, I.; Fratzl, P. The Role of Wheat Awns in the Seed Dispersal Unit. Science 2007, 316, 884-886.

(19) Ma, M.; Guo, L.; Anderson, D. G.; Langer, R. Bio-Inspired Polymer Composite Actuator and Generator Driven by Water Gradients. Science 2013, 339, 186-189.

(20) Beebe, D.; Moore, J.; Bauer, J.; Yu, Q.; Liu, R.; Devadoss, C.; Jo, B. Functional Hydrogel Structures for Autonomous Flow Control inside Microfluidic Channels. Nature 2000, 404, 588590.

(21) Zhao, Q.; Dunlop, J. W. C.; Qiu, X.; Huang, F.; Zhang, Z.; Heyda, J.; Dzubiella, J.; Antonietti, M.; Yuan, J. An Instant Multi-Responsive Porous Polymer Actuator Driven by Solvent Molecule Sorption. Nat. Commun. 2014, 5, 4293.

(22) Sidorenko, A.; Krupenkin, T.; Taylor, A.; Fratzl, P.; Aizenberg, J. Reversible Switching of Hydrogel-Actuated Nanostructures into Complex Micropatterns. Science 2007, 315, 487-490.

(23) Zhang, L.; Chizhik, S.; Wen, Y.; Naumov, P. Directed Motility of Hygroresponsive Biomimetic Actuators. Adv. Funct. Mater. 2016, 26, 1040-1053.

(24) Zhang, L.; Desta, I.; Naumov, P. Synergistic Action of Thermoresponsive and Hygroresponsive Elements Elicits Rapid and Directional Response of a Bilayer Actuator. Chem. Commun. 2016, 52, 5920-5923.

(25) Ma, Y.; Zhang, Y.; Wu, B.; Sun, W.; Li, Z.; Sun, J. Polyelectrolyte Multilayer Films for Building Energetic Walking Devices. Angew. Chemie Int. Ed. 2011, 50, 6254-6257.

(26) Dai, M.; Picot, O. T.; Verjans, J. M. N.; de Haan, L. T.; Schenning, A. P. H. J.; Peijs, T.; Bastiaansen, C. W. M. Humidity-Responsive Bilayer Actuators Based on a Liquid-Crystalline Polymer Network. ACS Appl. Mater. Interfaces 2013, 5, 4945-4950. 
(27) Chen, X.; Goodnight, D.; Gao, Z.; Cavusoglu, A. H.; Sabharwal, N.; DeLay, M.; Driks, A.; Sahin, O. Scaling up Nanoscale Water-Driven Energy Conversion into Evaporation-Driven Engines and Generators. Nat. Commun. 2015, 6, 7346.

(28) Takashima, Y.; Hatanaka, S.; Otsubo, M.; Nakahata, M.; Kakuta, T.; Hashidzume, A.; Yamaguchi, H.; Harada, A. Expansion-Contraction of Photoresponsive Artificial Muscle Regulated by Host-Guest Interactions. Nat. Commun. 2012, 3, 1270.

(29) Stoychev, G.; Turcaud, S.; Dunlop, J. W. C.; Ionov, L. Hierarchical Multi-Step Folding of Polymer Bilayers. Adv. Funct. Mater. 2013, 23, 2295-2300.

(30) Biener, J.; Wittstock, A.; Zepeda-Ruiz, L. A.; Biener, M. M.; Zielasek, V.; Kramer, D.; Viswanath, R. N.; Weissmüller, J.; Bäumer, M.; Hamza, A. V. Surface-Chemistry-Driven Actuation in Nanoporous Gold. Nat. Mater. 2009, 8, 47-51.

(31) Cheng, C.; Ngan, A. H. W. Reversible Electrochemical Actuation of Metallic Nanohoneycombs Induced by Pseudocapacitive Redox Processes. ACS Nano 2015, 9, 3984-3995.

(32) Liang, J.; Huang, L.; Li, N.; Huang, Y.; Wu, Y.; Fang, S.; Oh, J.; Kozlov, M.; Ma, Y.; Li, F.; Baughman, R.; Chen, Y. Electromechanical Actuator with Controllable Motion, Fast Response Rate, and High-Frequency Resonance Based on Graphene and Polydiacetylene. ACS Nano 2012, 6, 4508-4519.

(33) Sharifi, P.; Marmiroli, B.; Sartori, B.; Cacho-nerin, F.; Amenitsch, H.; Paris, O.; Leoben, M.; Forschung, L.; Source, D. L.; Science, H. Humidity Driven Deformation of Ordered Mesoporous Silica Films. Bioinspired, Biomim. Nanobiomaterials 2014, 3, 1-13.

(34) Boudot, M.; Gaud, V.; Louarn, M.; Selmane, M.; Grosso, D. Sol-Gel Based Hydrophobic Antireflective Coatings on Organic Substrates: A Detailed Investigation of Ammonia Vapor Treatment (AVT). Chem. Mater. 2014, 26, 1822-1833.

(35) Boissiere, C.; Grosso, D.; Lepoutre, S.; Nicole, L.; Bruneau, A. B.; Sanchez, C. Porosity and Mechanical Properties of Mesoporous Thin Films Assessed by Environmental Ellipsometric Porosimetry. Langmuir 2005, 21, 12362-12371.

(36) Reichenauer, G.; Scherer, G. W. Nitrogen Adsorption in Compliant Materials. J. Non. Cryst. Solids 2000, 277, 162-172.

(37) Reyssat, E.; Mahadevan, L. Hygromorphs: From Pine Cones to Biomimetic Bilayers. J. R. Soc. Interface 2009, 6, 951-957.

(38) Wang, K.; Cheng, C.; Cardona, E.; Guan, J.; Liu, K.; Wu, J. Performance Limits of Microactuation with Vanadium Dioxide as a Solid Engine. ACS Nano 2013, 7, 2266-2272.

(39) Timoshenko, S. Analysis of Bi-Metal Thermostats. J. Opt. Soc. Am. 1925, 11, 233.

(40) Brinker, C. J.; Lu, Y.; Sellinger, A.; Fan, H. Evaporation-Induced Self-Assembly: Nanostructures Made Easy. Adv. Mater. 1999, 11, 579-585.

(41) Clark, R. P. The Thermal Decomposition of Poly-4,4'-oxydiphenylene Pyromellitimide. Thermochim. Acta 1973, 6, 473-480.

(42) C.J. Brinker and G.W. Scherer. Sol Gel Science: The Physics and Chemistry of Sol-Gel Processing; Academic Press, NY, 1990.

(43) Scherer, G. W. Sintering of Sol-Gel Films. J. Sol-Gel Sci. Technol. 1997, 8, 353-363.

(44) Kozuka, H. Stress Evolution on Gel-to-Ceramic Thin Film Conversion. J. Sol-Gel Sci. Technol. 2006, 40, 287-297.

(45) Syms, R. R. a.; Holmes, a. S. Deposition of Thick Silica-Titania Sol-Gel Films on Si Substrates. J. Non. Cryst. Solids 1994, 170, 223-233.

(46) Parrill, T. Heat Treatment of Spun-on Acid-Catalyzed Sol-Gel Silica Films. J. Mater. Res. 1993, 9, 
723-730.

(47) Fardad, M. A.; Yeatman, E. M.; Dawnay, E. J. C.; Green, M.; Horowitz, F. Effects of $\mathrm{H} 2 \mathrm{O}$ on Structure of Acid-Catalysed SiO2 Sol-Gel Films. J. Non. Cryst. Solids 1995, 183, 260-267.

(48) Kozuka, H. On Ceramic Thin Film Formation from Gels: Evolution of Stress, Cracks and Radiative Striations. J. Ceram. Soc. Japan 2003, 111, 624-632.

(49) Stoney, G. G. The Tension of Metallic Films Deposited by Electrolysis. Proc. R. Soc. A Math. Phys. Eng. Sci. 1909, 82, 172-175.

(50) Janssen, G. C. a. M.; Abdalla, M. M.; van Keulen, F.; Pujada, B. R.; van Venrooy, B. Celebrating the 100th Anniversary of the Stoney Equation for Film Stress: Developments from Polycrystalline Steel Strips to Single Crystal Silicon Wafers. Thin Solid Films 2009, 517, 18581867.

(51) Niklasson, G. a; Granqvist, C. G.; Hunderi, O. Effective Medium Models for the Optical Properties of Inhomogeneous Materials. Appl. Opt. 1981, 20, 26-30.

(52) Crepaldi, E. L.; Soler-Illia, G. J. de A. A.; Grosso, D.; Cagnol, F.; Ribot, F.; Sanchez, C. Controlled Formation of Highly Organized Mesoporous Titania Thin Films: From Mesostructured Hybrids to Mesoporous Nanoanatase TiO 2. J. Am. Chem. Soc. 2003, 125, 9770-9786.

(53) Xie, K.; Zhang, S. Y.; Liu, J. G.; He, M. H.; Yang, S. Y. Synthesis and Characterization of Soluble Fluorine-Containing Polyimides Based on 1,4-bis(4-Amino-2-Trifluoromethylphenoxy)benzene. J. Polym. Sci. Part A Polym. Chem. 2001, 39, 2581-2590.

(54) Samuel, J.; Brinker, C. J.; Douglas Frink, L. J.; van Swol, F. Direct Measurement of Solvation Forces in Complex Microporous Media: A New Characterization Tool. Langmuir 1998, 14, 2602-2605.

(55) BANGHAM, D. H.; FAKHOURY, N. The Expansion of Charcoal Accompanying Sorption of Gases and Vapours. Nature 1928, 122, 681-682.

(56) Meehan, F. T. The Expansion of Charcoal on Sorption of Carbon Dioxide. Proceedings of the Royal Society A: Mathematical, Physical and Engineering Sciences, 1927, 115, 199-207.

(57) Ravikovitch, P. I.; Neimark, A. V. Density Functional Theory Model of Adsorption Deformation. Langmuir 2006, 22, 10864-10868.

(58) Balzer, C.; Braxmeier, S.; Neimark, A. V.; Reichenauer, G. Deformation of Microporous Carbon during Adsorption of Nitrogen, Argon, Carbon Dioxide, and Water Studied by in Situ Dilatometry. Langmuir 2015, 31, 12512-12519.

(59) Douglas Frink, L. J.; Van Swol, F. Stress Isotherms of Porous Thin Materials: Theoretical Predictions from a Nonlocal Density Functional Theory. Langmuir 1999, 15, 3296-3301.

TOC. 


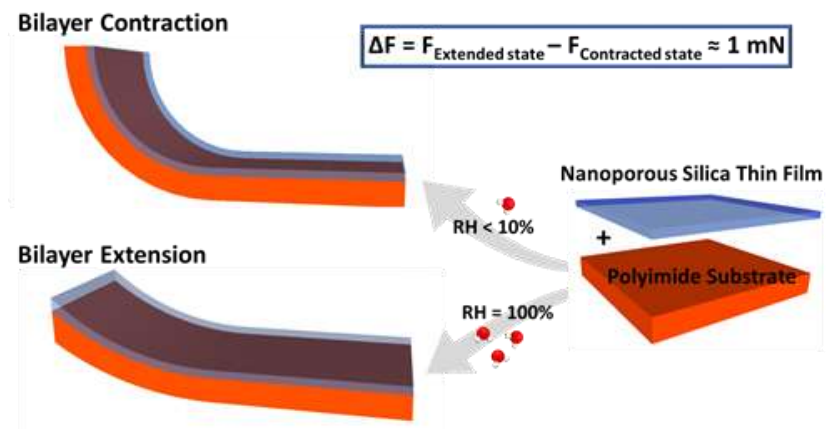

